\title{
CHELERYTHRINE PREVENTS MICE LIVER FIBROGENESIS INJURY VIA REGULATION OF TGFB/SMADS SIGNAL PATHWAY
}

\author{
XIAOMING LI ${ }^{1}$, HONGLIAN ZHANG ${ }^{2}$, and XIAOLI WANG ${ }^{2 *}$
}

${ }^{1}$ The Institute of Medicine, Qiqihar Medical University, Qiqihar, China

${ }^{2}$ School of Pharmacy, Qiqihar Medical University, Qiqihar, China

\begin{abstract}
The aim of the study was to investigate the effect of chelerythrine (CHE) anti-liver fibrosis in mice and the underlying mechanism through regulation of TGF $\beta /$ smads signaling pathway. Here, we demonstrate that chelerythrine suppressed the factor expression of $\mathrm{CCI}_{4}$ and $\mathrm{BDL}$ induced liver injury markers ALT, AST, HA, Hyp, as well as HSCs activation and ECM deposition. Mechanistically, chelerythrine promoted the concentration of GSH and reduced the content of ROS. Simultaneously, chelerythrine inhibited TGF $\beta$ secretion and restrained T $\beta$ RI, T $\beta$ RII, smad 3, smad4 expression. Furthermore, chelerythrine prevented the activation of HSCs through TGF $\beta /$ smads signaling pathway. Collectively, the data suggested chelerythrine inhibited extracellular matrix formation and alleviated $\mathrm{CCl}_{4}$ and $\mathrm{BDL}$ mice liver fibrosis, preventing activation of HSCs, via suppressing oxidative stress and the TGF $\beta / \mathrm{smads}$ signaling pathway.
\end{abstract}

Keywords: chelerythrine, hepatic fibrosis, hepatic stellate cells, transforming growth factor- $\beta$, smads

\begin{abstract}
Abbreviation: $\mathrm{CHE}$ : chelerythrine, $\mathrm{CCl}_{4}$ : carbon tetrachloride, BDL: bile duct ligation, ALT: alanine aminotransferase, AST: aspartate aminotransferase, HA: hyaluronic acid, Hyp: hepatic hydroxyproline, ROS: reactive oxygen species, GSH: glutathione, HE: hematoxylin-eosin, RT-PCR: real-time reverse transcription-polymerase chain reaction, HSCs: hepatic stellate cell lines, FN: fibronectin, ECM: extracellular matrix
\end{abstract}

Hepatic fibrosis is chronic liver damage by different causes (1). Under the present circumstances, normal hepatic tissue is replaced with collagen-rich extracellular matrix (ECM) and, if left untreated, results in cirrhosis (2). It is a common pathological basis for various chronic liver diseases and a necessary stage for liver cirrhosis and liver cancer (3). Studies have shown: in pathological conditions, hepatic fibrosis is characterized by the imbalance between deposition and degradation of extracellular matrix (ECM) (4). Several studies have shown that hepatic fibrosis is a reversible disease, therefore finding an effective treatment would probably prevent or reverse the fibrotic process in the liver (5). Meanwhile, various current research has demonstrated, activation of hepatic star cells (HSCs) plays an important role in liver fibrosis. Transforming growth factor $\beta 1$ (TGF $\beta 1$ ) is one of the strongest profibrotic cytokine (6), stimulating and inducing the activation and proliferation of hepatic star cells (HSCs). Simultaneously, it is a key factor that promotes excessive deposition of extracellular matrix
(ECM), Moreover, the smads family proteins are key proteins in TGF $\beta$ signaling, and different smads family proteins play different roles in signal transduction (7). Therefore, how to inhibit activation of hepatic star cells(HSCs) and excessive deposition of extracellular matrix (ECM) and then reverse liver fibrosis and prevent liver cirrhosis and liver cancer has become the top priority for medical researchers.

Chelerythrine (CHE) is a natural quaternary benzophenanthridinium alkaloid which is a constituent of the herb of Chelidonium majus L. It displays a great diversity of bioactivities like antitumor, antiinflammatory, and antimicrobial. The current study has evaluated that chelerythrine is used in the treatment of liver fibrosis through regulating ALT activity (8). In this study, we investigated the hypothesis that chelerythrine might protect the liver from $\mathrm{CCl}_{4}$ induced and BDL-induced fibrogenesis by anti-oxidization and inhibiting the deposition of ECM and activation of HSC via modulating TGF $\beta 1 /$ smad signaling pathways. The results of this report support our hypothesis and provide novel insights into the

* Corresponding author: e-mail: ccwxl@qmu.edu.cn 
mechanisms of the antifibrogenic effect of chelerythrine in the protection of the liver.

\section{MATERIAL AND METHODS}

\section{Reagents, antibodies, and instruments}

AST, ALT, HA, Hyp, and Van Gieson kits were obtained from Beijing Solarbio Science \& Technology Co., Ltd. Reactive oxygen species (ROS) and glutathione (GSH) Assay Kit was purchased from Jiancheng Bioengineering Institute (Nanjing, China). A protein extraction kit was obtained from Beytime Biotechnology. TRIzol Reagent was obtained from Ambion Company. The $\alpha$-SMA,Col I, Fibronectin, TGF $\beta$, T $\beta$ RI, T $\beta$ RII, smad 3, smad4, smad7 primer were obtained from Sangon Biotech (Shanghai, China). The glyceraldehyde 3-phosphate dehydrogenase (GAPDH) antibody and the secondary antibodies were obtained from Cell Signaling Technology. The $\alpha$-SMA, Col I, Fibronectin, TGF $\beta$, T $\beta$ RI, T $\beta$ RII, smad3, Smad4 antibodies were obtained from Cell Signaling Technology. The smad7 antibody was obtained from Santa Cruz Company. Chelerythrine was purchased from National Institutes for Food and Drug Control (Purity: 98\% by HPLC).

\section{Animals}

This study was approved by the Animal Care and Use Committee of Qiqihar Medical University (Approval number (Qi) Lun Shen [2016] 02.). A total of 80 pathogen-free male C57BL/6N (SPF) mice (7-8 weeks old, weight range: $35.0 \pm 2.0 \mathrm{~g}$ ) were employed in the study. The animals were obtained from the Nanjing Biomedical Research Institute of Nanjing University (Nanjing, Jiangsu, China). They were housed in separate sterile ventilated cages in a specific pathogen-free room with a $12 \mathrm{~h}$ light and $12 \mathrm{~h}$ dark schedule and fed autoclaved chow and water. These experimental animals were weighed every 7 days in order to adjust the $\mathrm{CCI}_{4}$ and chelerythrine doses.

\section{Cell culture and treatment}

HSCs, a mouse hepatic stellate cell line, were purchased from the Cancer Institute and Hospital of CAMS (Beijing, China). HSCs were cultured in Dulbecco's modified Eagle medium supplemented with $10 \%$ fetal calf serum and $1 \%$ penicillin-streptomycin at $37^{\circ} \mathrm{C}$ in an atmosphere containing $5 \%$ $\mathrm{CO}_{2}$. Based on our previous research, HSCs were activated by TGF $\beta$. In further experiments, $20 \mu \mathrm{g} / \mathrm{L}$ of TGF $\beta$ for 24 hours was used. The activated HSCs were added with chelerythrine at a concentration of $3.6 \mu \mathrm{M}$ and cultured for 24 hours.

\section{Establishment of a mouse model with hepatic fibrogenesis caused by CCI4}

Total C57BL/6N mice (40 mice) were divided into the following four groups ( $\mathrm{n}=10$ per group): normal group, $\mathrm{CCI}_{4}$-model group, chelerythrine $(20 \mathrm{mg} / \mathrm{kg})+\mathrm{CCI}_{4}$ treated-group, chelerythrine $(40 \mathrm{mg} / \mathrm{kg})+\mathrm{CCI}_{4}$-treated group. Throughout the experiment, the normal group drank and ate normally, the $\mathrm{CCI}_{4}$ model group and chelerythrine treated groups were administered olive oil and $\mathrm{CCI}_{4}$. The first time, the other three groups $\left(\mathrm{CCI}_{4}\right.$ model group and chelerythrine treated-groups) were administered $\mathrm{CCI}_{4}$ via intraperitoneal injection $(3 \mathrm{~mL} / \mathrm{kg}$ body weight), the other times, which were administered $50 \% \mathrm{CCI}_{4}$ and olive oil via intraperitoneal injection (two times /week, 8weeks) (9-10). In the fifth week, the other two treated groups were treated with $20 \mathrm{mg}$ and $40 \mathrm{mg}$ chelerythrine via gavage per $\mathrm{Kg}$ of body weight/day for fourteen weeks. After fourteen weeks, mice were sacrificed after being anesthetized by ip. pentobarbital $(50 \mathrm{mg} / \mathrm{kg})$. Blood was collected immediately after the animals of each group were sacrificed. Then, serum was separated by centrifugation within $1 \mathrm{~h}$ and stored at $-20^{\circ} \mathrm{C}$ until analyzed. A small portion of the liver was fixed with $10 \%$ formalin for histopathological observation, and the rest was stored in liquid nitrogen for extraction of proteins and RNA.

\section{Establishment of a mouse model with hepatic fibrogenesis caused by BDL}

In the BDL model, mice were anesthetized with $0.1 \mathrm{~mL} / 10 \mathrm{~g} \mathrm{4} \%$ chloral hydrate intraperitoneal injection after fasting 24 hours. Then, mice were placed on a sterile, heated table, the mouse's abdominal cavity was opened, the bile duct was exposed, and then ligated with silk. In sham groups, the cavity was only opened without doing anything (11-12). After bile duct ligation, chelerythrine was administered to mice by gavage for two weeks, and two weeks later, blood samples and liver tissue were collected for further experiments.

\section{Biochemical parameters}

Activities of alanine transaminase (ALT), aspartate aminotransferase (AST), and Hyaluronic acid (HA) in serum were measured by routine laboratory methods using a 7170-automatic biochemistry analyzer (Tokyo, Japan).

\section{Determination \\ of the hepatic hydroxyproline content}

The content of hepatic hydroxyproline was determined by the hydroxyproline kit following 
Table 1. The primer sequences for RT-PCR in this study.

\begin{tabular}{|c|c|c|}
\hline \multicolumn{2}{|c|}{ Gene } & Primer sequence $\left(5^{\prime}-3^{\prime}\right)$ \\
\hline \multirow{2}{*}{ Col-I } & Forward & GAG TGA GGCCAC GCA TGA \\
\hline & Reverse & AGC CGG AGG TCC ACA AAG \\
\hline \multirow{2}{*}{ Fibronectin } & Forward & TGT CAC CCA CCA CCT TGA \\
\hline & Reverse & CTG ATTGTT CTT CAG TGC GA \\
\hline \multirow{2}{*}{$\alpha$-SMA } & Forward & CCG AGA TCTCAC CGA CTA CC \\
\hline & Reverse & TCC AGA GCG ACA TAG CAC AG \\
\hline \multirow{2}{*}{ TIMP-1 } & Forward & GCAACTCGGACCTGGTCATAA \\
\hline & Reverse & CGGCCCGTGATGAGAAACT \\
\hline \multirow{2}{*}{ MMP-2 } & Forward & CAAGTTCCCCGGCGATGTC \\
\hline & Reverse & TTCTGGTCAAGGTCACCTGTC \\
\hline \multirow{2}{*}{ TGF- $\beta 1$} & Forward & GTG TGG AGC AAC ATG TGG AAC TCT A \\
\hline & Reverse & CGCTGA AC GAA AGC CCT GTA \\
\hline \multirow{2}{*}{ TGF $\beta$ RI } & Forward & GCT GAC ATC TAT GCA ATG GGC TTA \\
\hline & Reverse & AGG CAA CTG GTA GTC TTC GTG GA \\
\hline \multirow{2}{*}{ TGF $\beta$ RII } & Forward & GCG ATC TAACCT GTT GCC TGT G \\
\hline & Reverse & GGG CCA TGT ATC TCG CTGTTC \\
\hline \multirow{2}{*}{ Smad3 } & Forward & TGGCTACCTGAGTGAAGATGG \\
\hline & Reverse & AGTTATTGTGTGCTGGGGACA \\
\hline \multirow{2}{*}{ Smad4 } & Forward & TGGAAG TAG GAC TGC ACC ATA CAC A \\
\hline & Reverse & AGC AAT GGAACA CCA ATA TTC AGG A \\
\hline \multirow{2}{*}{ Smad7 } & Forward & AGCAAGAGTCAGCACTGCCAAG \\
\hline & Reverse & TGACAACTGAAATGCTGATCCAAAG \\
\hline \multirow{2}{*}{ GAPDH } & Forward & GAC AAC TTT GGC ATC GTG GA \\
\hline & Reverse & ATG CAGGGA TGA TGT TCT GG \\
\hline
\end{tabular}

the protocol provided by the manufacturer. Results were expressed as micrograms of hydroxyproline per gram of hepatic tissue.

\section{Determination of mice serum ROS and GSH concentration}

The absorbance value of mice serum ROS and GSH were determined by using the colorimetric method. On the basis of the absorbance value, the concentration of ROS and GSH were assayed by computer software.

\section{Liver histopathology}

Liver tissues were fixed in $10 \%$ neutral buffered formalin and embedded in paraffin. Preparing $4 \mu \mathrm{m}$ thick paraffin sections of liver tissue and then performing Hematoxylin-eosin (HE) and Sirus Red staining using a standard method. Photographs were analyzed quantitatively with the IMAGEPRO PLUS 6.0 to judge and evaluate the degree of liver fibrosis.

\section{RT-PCR Assay}

Total RNA was extracted from frozen liver tissue and HSCs according to the instructions of the RNAiso Reagent kit. cDNA was synthesized with SYBR ExScript ${ }^{\mathrm{TM}}$ RT-PCR kit according to the protocol provided by the manufacturer. The relative levels of the target genes were normalized with the internal control Glyceraldehyde phosphate dehydrogenase (GAPDH). mRNA-fold change relative to GAPDH was calculated with the comparative $\mathrm{Ct}$ method of $2^{-\Delta \Delta \mathrm{Ct}}$.

The primer sequences for RT-PCR in this study are listed in Table 1.

\section{Western blot analyses}

Total proteins were extracted from liver tissues and HSCs using a Cytoplasmic Protein Extraction kit according to the protocol provided by the manufacturer. The protein contents were tested with a BCA assay kit according to the protocol provided by the manufacturer. In this report, SDS-PAGE 
was used to analyze the target proteins, first, which were analyzed by adding primary antibodies for 4 hours, and then, a secondary antibody for 2 hours (horseradish peroxidase-conjugated). The blots were scanned using an ECL Western blotting kit as recommended by the manufacturer. GAPDH was used as an internal control. The relative protein content of each lane was normalized with the internal control. Band intensities were determined using an AlphaImager ${ }^{\mathrm{TM}} 2200$ adopting the SpotDenso function of AlphaEaseFC ${ }^{\text {TM }}$ Software version 3.1.2 (Witec, Littau, Switzerland).

\section{Statistical analysis}

The data were expressed as the mean \pm SD. ANOVA was used to evaluate the difference among multiple groups followed by a post hoc test (StudentNewman-Keuls) for quantitative data, and the RIDIT test was used for statistical analysis of qualitative data. The data were analyzed using SPSS 17.0 software (SPSS Inc., Chicago, IL, USA), and $\mathrm{P}<0.05$ was considered statistically significant.

\section{RESULTS}

\section{Effect of chelerythrine on the serum concentration of ROS and GSH in the CCI4 and BDL mice model}

In order to clarify the effect of chelerythrine on oxidative stress factors in $\mathrm{CCI}_{4}$ and BDL model mice, we used a colorimetric method to determine the serum concentration of ROS and GSH in each group. Figure 1 have shown, compared with the control (sham) group, the concentration of ROS was significantly up-regulated, the concentration of GSH was down-regulated in $\mathrm{CCI}_{4}$ and $\mathrm{BDL}$ model groups. After intervention with chelerythrine, the above changes of ROS and GSH were reversed in chelerythrine dose-dependent.

\section{Chelerythrine inhibited serum activities of ALT, AST, HA in the CCI4 and BDL mice model}

To investigate whether chelerythrine was protective against liver injury, we examined changes in ALT, AST, and HA levels in serum. Figure 2 showed, compared with those in the normal control, the

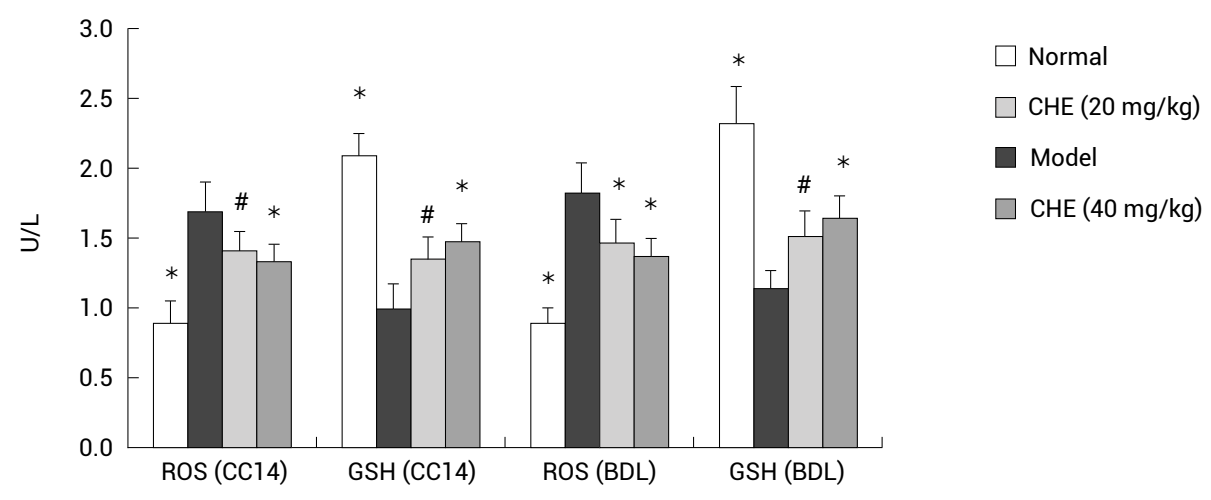

Figure 1. Effect of chelerythrine on the concentration of ROS and GSH in $\mathrm{CCI}_{4}$ and BDL induced model mice. Mean $\pm \mathrm{SD}$. $\mathrm{n}=3$. $* \mathrm{P}<0.01, \# \mathrm{P}<0.05$ vs Model group.
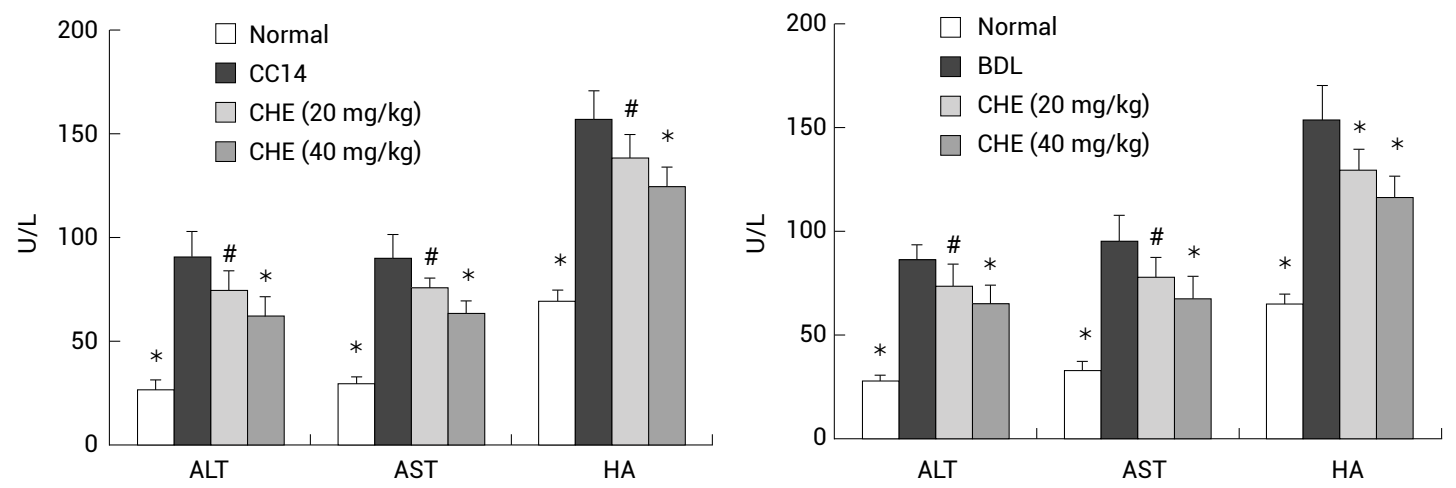

Figure 2. The protective effect of chelerythrine on $\mathrm{CCI}_{4}$ and $\mathrm{BDL}$ induced liver fibrosis mice. a) chelerythrine reduced serum AST, ALT, HA content in $\mathrm{CCI}_{4}$ model mice. b) chelerythrine reduced serum AST, ALT, HA content in BDL model mice. Mean $\pm \mathrm{SD}$. $\mathrm{n}=3$, $* \mathrm{P}<0.01, \# \mathrm{P}<0.05$ vs Model group Mean $\pm \mathrm{SD}, \mathrm{n}=3, * \mathrm{P}<0.01, \# \mathrm{P}<0.05$ vs Model group. 


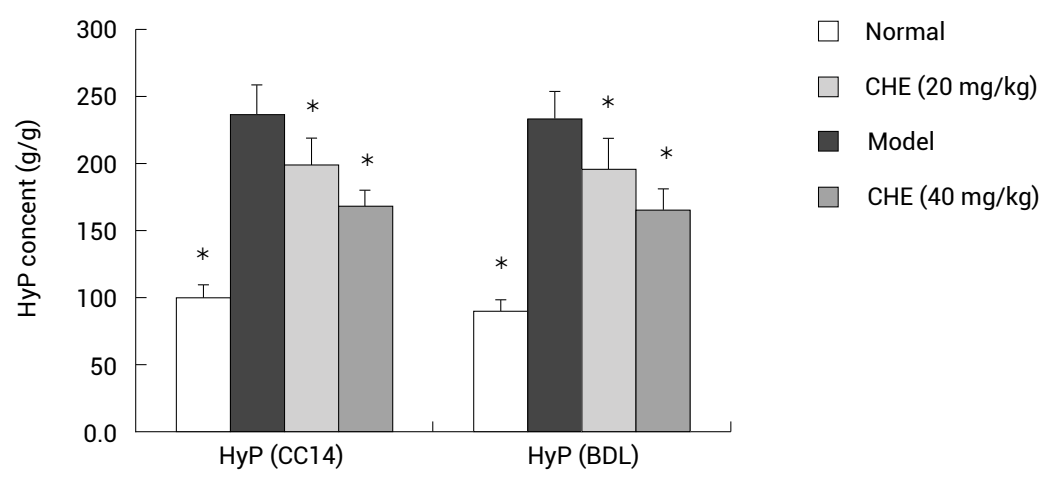

Figure 3. Chelerythrine reduced the content of liver tissue hyp in $\mathrm{CCI}_{4}$ and BDL model mice. Mean $\pm \mathrm{SD}, \mathrm{n}=3$, $* \mathrm{P}<0.01$, vs Model group.

contents of serum ALT, AST, HA were significantly higher in the $\mathrm{CCI}_{4}$ and BDL mice model group. In chelerythrine-treated groups, the contents of serum ALT, AST, and HA were significantly reduced in a dose-dependent manner. The results indicated that chelerythrine can protect against liver damage caused by carbon tetrachloride and bile duct ligation.

\section{Chelerythrine reduced the content of hepatic hydroxyproline in the CCI4 and BDL mice model}

To further explore the protective effect of chelerythrine on the liver, the content of hepatic hydroxyproline was detected by quantitative analysis, As shown in Figure 3, Compared with the normal controls, the hepatic hydroxyproline content was significantly higher in the $\mathrm{CCI}_{4}$ and BDL mice model. The content of hydroxyproline in the liver was significantly reduced after treatment with chelerythrine, especially in the high-dose group.

\section{Protective effects of chelerythrine on alteration of histopathology}

The effects of chelerythrine on liver and antifibrosis were evaluated by histochemical analysis. Representative liver tissue sections are shown in Figure 4, Hematoxylin-eosin (HE) and Sirus Red staining showed, in the liver tissue of the model group, the area of collagen fibers increased significantly. Oral administration of chelerythrine daily, chelerythrine significantly reduced liver damage and collagen staining area. Chelerythrine had a protective effect on liver injury, and the high-dose group was better than the low-dose group.

\section{Chelerythrine inhibited hepatic stellate activation and deposition of extracellular matrix}

Liver fibrosis is characterized by activation of hepatic stellate cells and deposition of extracellular matrix. As shown in Figure 5a, b, c, d, e, f, RTPCR, and western blotting analyses showed Col-I, $\alpha$-SMA, and TIMP-1 expressions were significantly increased in BDL-CCI ${ }_{4}$-model mice compared with mice in the sham and normal groups, and both were decreased in the group treated by chelerythrine. The expression of MMP-2 was reversed, the expression of MMP-2 was decreased in the model group, and, was increased by chelerythrine treatment, in a dosedependent manner.

To mimic the liver fibrosis induced by carbon tetrachloride and BDL, in vitro, we investigated whether chelerythrine inhibits the activation of hepatic stellate cells. The RT-PCR results were consistent with the western blotting shown in Figure $5 \mathrm{~g}$, Chelerythrine inhibits the activation of hepatic stellate cells in a concentration-dependent manner. Western blotting results show in Figure 5h, i, chelerythrine decreased the proteins expression of $\alpha$-SMA, Col-I, and fibronectin, in hepatic stellate cells. Taken together, these results show that Chelerythrine inhibits the activation of hepatic stellate cells and extracellular matrix deposition further against liver fibrosis.

\section{Chelerythrine inhibits CCI4 and BDL induced liver fibrosis in mice through TGFß/smad signaling pathway}

To investigate whether the TGF $\beta 1 / \mathrm{smad}$ signaling pathway was involved in the role of Chelerythrine in $\mathrm{CCI}_{4}$ and $\mathrm{BDL}$ induced liver fibrosis, and then TGF $\beta 1$, T $\beta$ R I, T $\beta$ R II, p-smad3, p-smad4, p-smad7 expression were evaluated. As indicated in Figure 6, $\mathrm{CCI}_{4}$ and BDL -induced hepatic fibrosis groups, TGF $\beta 1$, T $\beta$ R I, T $\beta$ R II mRNA expression increased, compared with the control group. Chelerythrine significantly decreased TGF $\beta 1$, T $\beta R$ I, T $\beta$ R II, smad 3 mRNA expression in liver tissue of mice with hepatic fibrosis (Figure 6a, b, c, d). Western blotting results showed that the 
a)
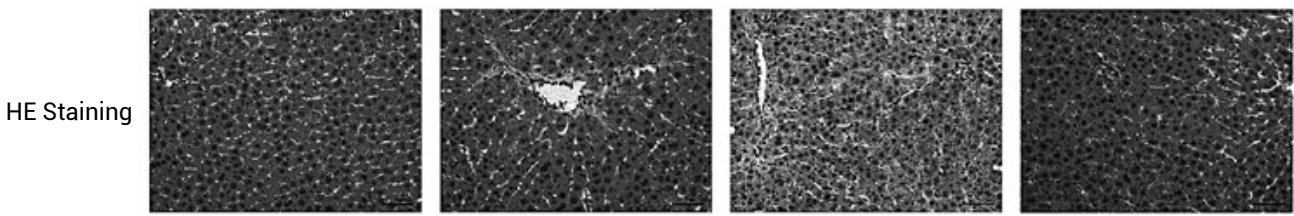

Sirius Red

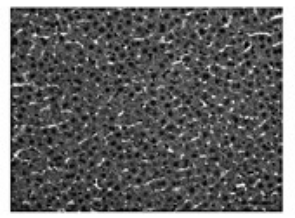

A

b)
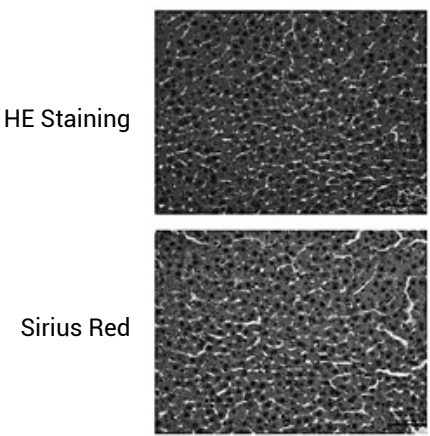

A

c)

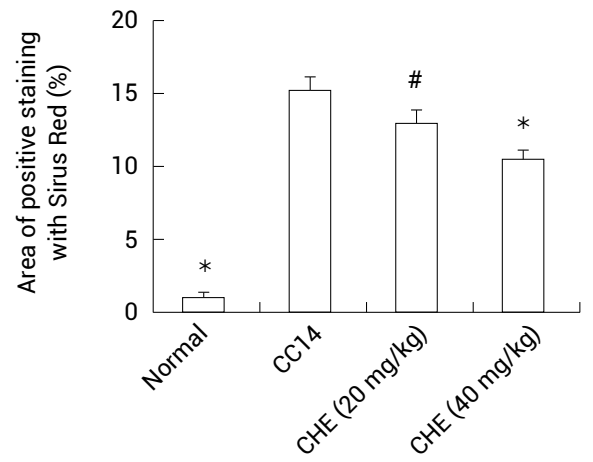

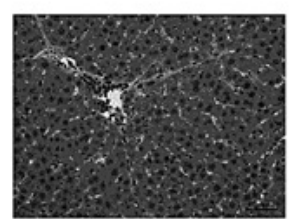

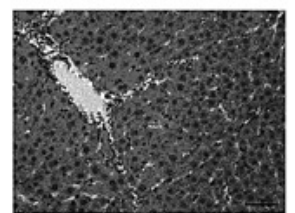

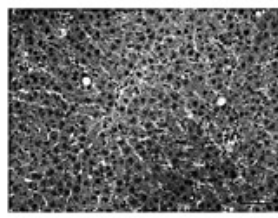

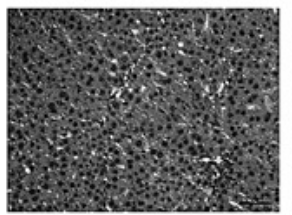

C
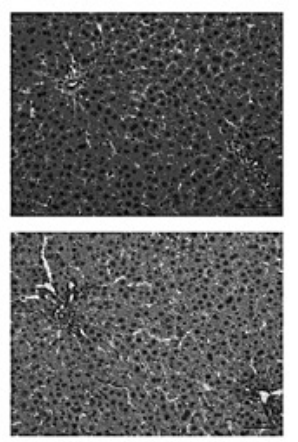

D

d)

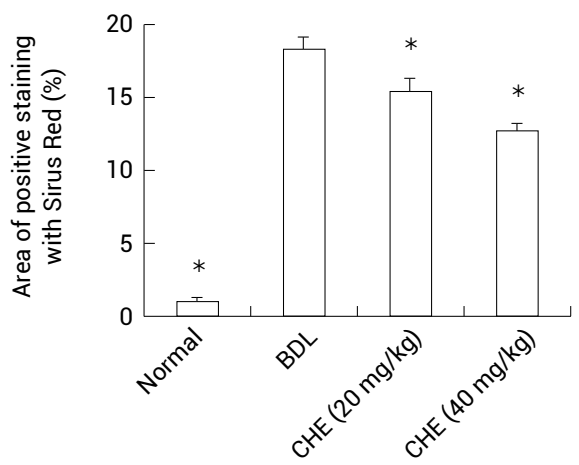

Figure 4. Protective effects of chelerythrine on alteration of histopathology. a) Hematoxylin-eosin (HE) and Sirus Red staining in $\mathrm{CCI}_{4}$ model mice (x 200), A) Normal group B) CCI4 model group C) low concentration group D) high concentration group. b) Hematoxylin-eosin (HE) and Sirus Red staining in BDL model mice (x 200), A) Normal group B) BDL model group C) low concentration group D) high concentration group. c) Semi-quantitative of Sirus Red staining $\left(\mathrm{CCI}_{4}\right.$ model). d) Semi-quantitative of Sirus Red staining(BDL model). ${ }^{*} \mathrm{P}<0.01$, \# $\mathrm{P}<0.05$ vs Model group.

expression of TGF $\beta$, T $\beta R$ I, T $\beta R$ II and phosphorylated smad3 were significantly increased in the two model groups, while the chelerythrine significantly reduced TGF $\beta 1$, T $\beta$ R I, T $\beta$ R II and expression, the phosphorylation level of smad3, but had no significant effect on the expression level of smad3 (Figure 6e, f, g, h).

Experiments further revealed that mRNA and protein levels of smad4 in liver tissues were significantly increased in $\mathrm{CCI}_{4}$ and BDL model mice. In contrast, protein and mRNA levels of smad4 in liver tissues from mice treated with chelerythrine were attenuated (Figure $6 \mathrm{~b}, \mathrm{~d}, \mathrm{e}, \mathrm{f}, \mathrm{g}$, h). However, compared with the model group, the expression of mRNA and protein of $\operatorname{smad} 7$ significantly increased in the chelerythrine groups (Figure 6b, d, e, f, g, h). Chelerythrine (20 mg/kg/d, $40 \mathrm{mg} / \mathrm{kg} / \mathrm{d}$ ) suppressed the $\mathrm{CCI}_{4}$, BDL induced 

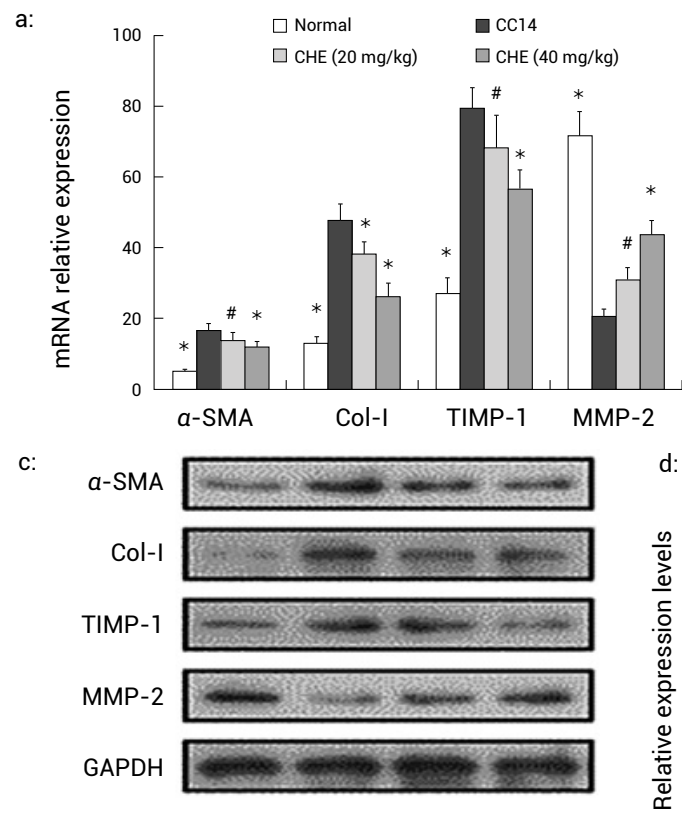

$$
\mathrm{CHE}(\mathrm{mg} / \mathrm{kg}) \quad-\quad \quad \quad-\quad 20 \quad 40
$$
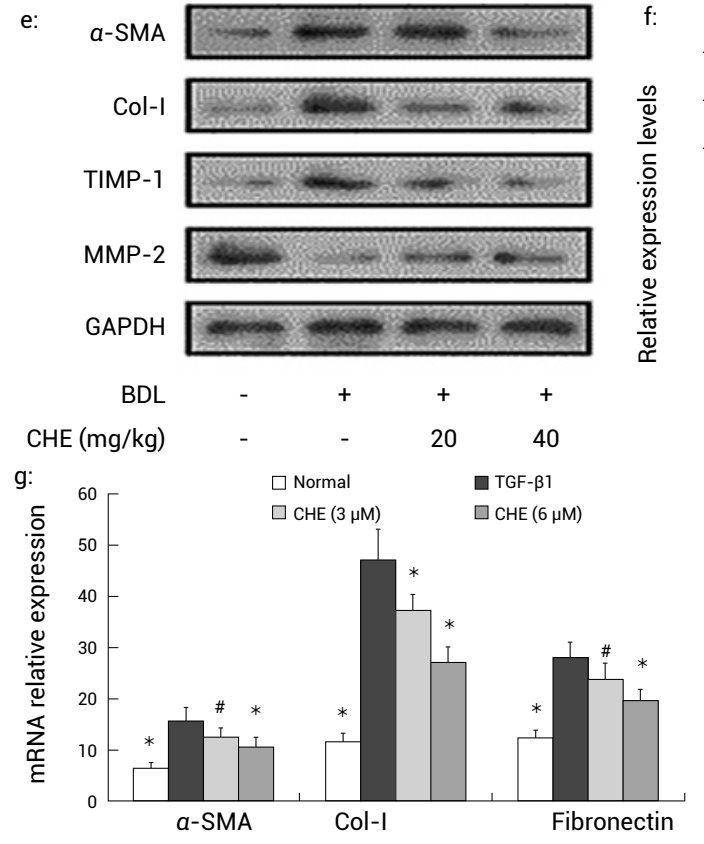

Figure 5. Chelerythrine inhibited hepatic stellate cell activation and ECM deposited in $\mathrm{CCI}_{4}$ and BDL-induced liver fibrosis mice. a) The effect of chelerythrine on $\alpha$-SMA, Col-I, TIMP-1, MMP-2 mRNA expression in $\mathrm{CCI}_{4}$-induced liver fibrosis mice. b) The effect of chelerythrine on $\alpha$-SMA, Col-I, TIMP-1, MMP-2 mRNA expression in BDL-induced liver fibrosis mice. c) The effect of chelerythrine on $\alpha$-SMA, Col-I, TIMP-1, MMP-2 protein expression in $\mathrm{CCI}_{4}$-induced liver fibrosis mice. d) Fold changes of $\alpha$-SMA, Col-I, TIMP-1, MMP2 expression from (c). e) The effect of chelerythrine on $\alpha$-SMA, Col-I, TIMP-1, MMP-2 protein expression in BDL-induced liver fibrosis mice. f) Fold changes of $\alpha$-SMA, Col-I, TIMP-1, MMP-2 expression from (e). g) The effect of Chelerythrine on $\alpha$-SMA, Col-I, FN mRNA expression in HSCs. h) The effect of chelerythrine on $\alpha$-SMA, Col-I, FN protein expression in HSCs. i) Fold changes of $\alpha$-SMA, Col-I, FN expression from (h). Mean $\pm \mathrm{SD}, \mathrm{n}=3, * \mathrm{P}<0.01, \# \mathrm{P}<0.05$ vs Model group.
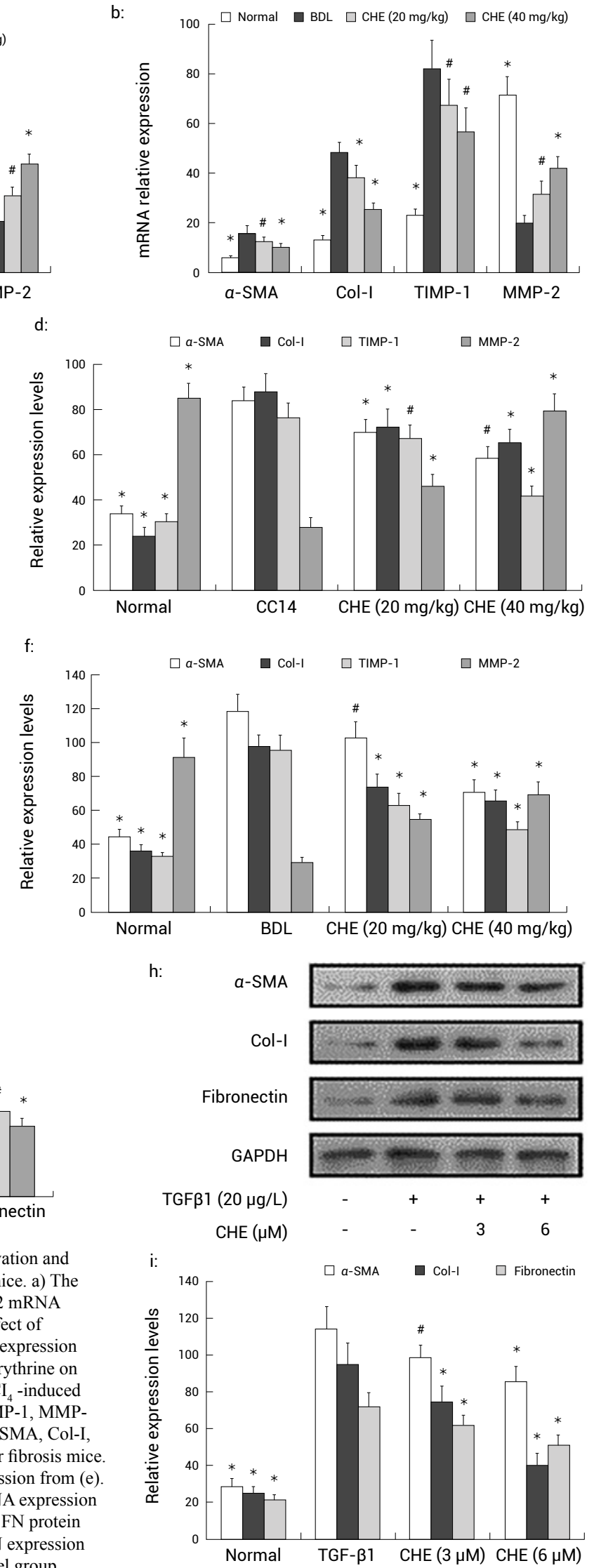

b:

TGF- $\beta 1 \quad$ CHE $(3 \mu \mathrm{M})$ CHE $(6 \mu \mathrm{M})$ 

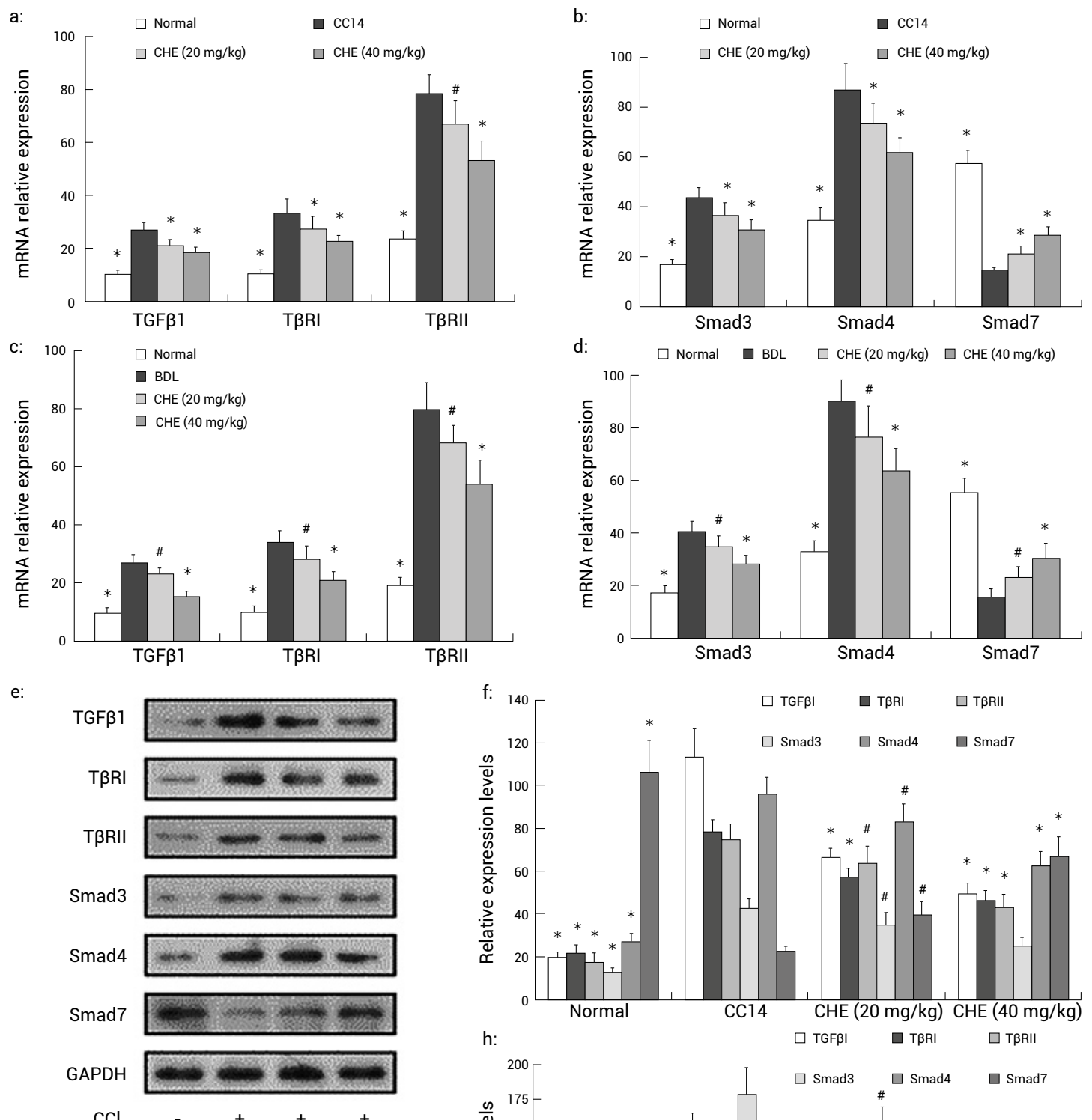

$\mathrm{CHE}(\mathrm{mg} / \mathrm{kg}) \quad$ - $\quad-\quad 20 \quad 40$

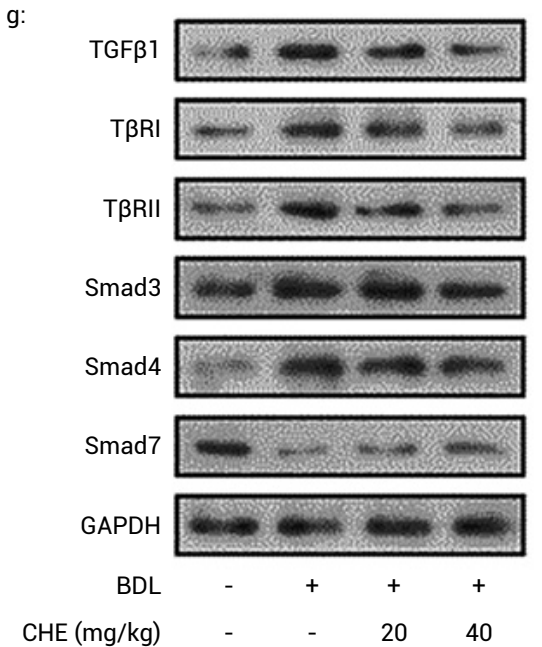

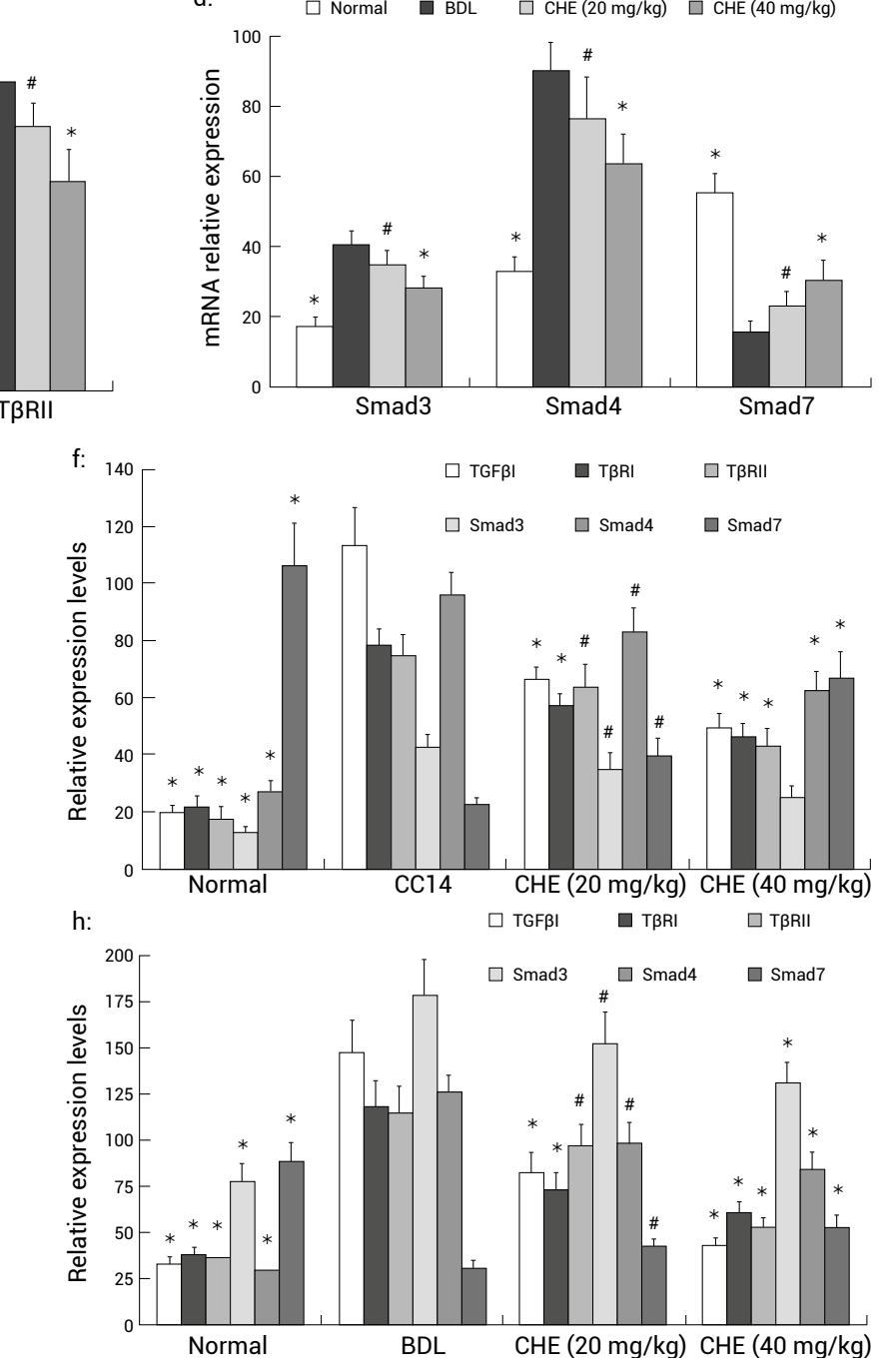

Figure 6. Chelerythrine prevented liver fibrosis by $\mathrm{CCI}_{4}$ and $\mathrm{BDL}$ induced through TGF $\beta /$ smad signaling pathway. a) The effect of chelerythrine on TGF $\beta$, T $\beta R$ I, TRR II mRNA expression in CCI induced model mice. b) The effect of chelerythrine on smad3, smad4, smad7 mRNA expression in $\mathrm{CCI}_{4}$ induced model mice. c) The effect of chelerythrine on TGF $\beta$, T $\beta R$ I, T $\beta R$ II mRNA expression in BDL induced model mice. d) The effect of chelerythrine on smad3, smad4, smad7 mRNA expression in BDL induced model mice. e) The effect of chelerythrine on TGF $\beta$, T $\beta R$ I, T $\beta R$ II, smad3, smad4, smad7 protein expression in $\mathrm{CCI}_{4}$ induced model mice.(f) Fold changes of TGF $\beta$, T $\beta R$ I, T $\beta \mathrm{R}$ II, smad3, smad4, smad7 expression from (e). g) The effect of chelerythrine on TGF $\beta$, TRR I, TRR II, smad3, smad4, smad7 protein expression in BDL induced model mice. h) Fold changes of TGF $\beta$, T $\beta R$ I, T $\beta R$ II, smad3, smad4, smad7 expression from (g). Mean $\pm \mathrm{SD}, \mathrm{n}=3,{ }^{*} \mathrm{P}<0.01, \# \mathrm{P}<0.05$ vs Model group. 

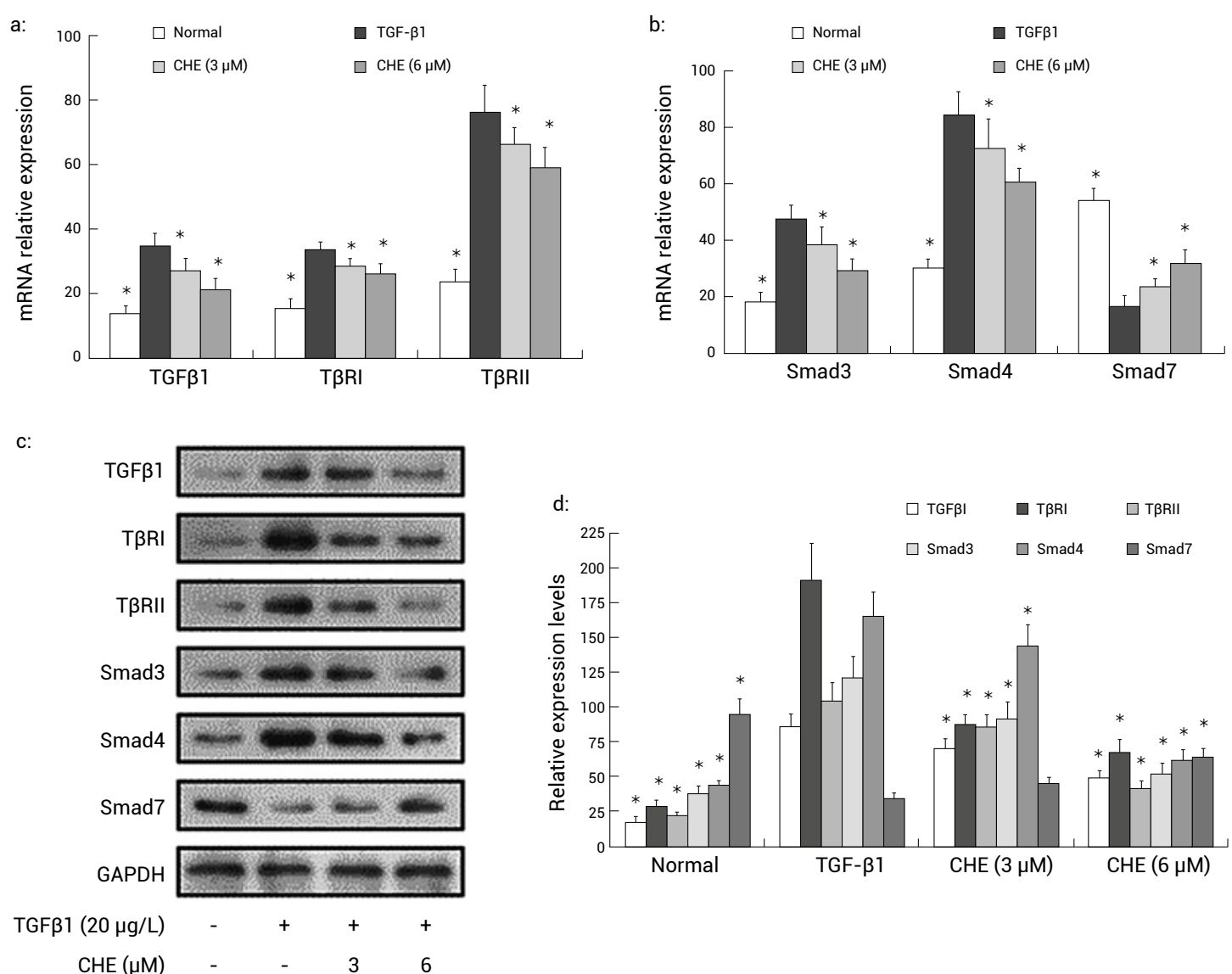

Figure 7. Chelerythrine inhibited hepatic stellate cell activation through TGF $\beta /$ smad signal pathway. a) The effect of chelerythrine on TGF $\beta$, T $\beta$ R I, T $\beta$ R II mRNA expression in HSCs. b) The effect of chelerythrine on smad3, smad4, smad7 mRNA expression in HSCs. c) The effect of chelerythrine on TGF $\beta$, T $\beta$ R I, T $\beta$ R II, smad3, smad4, smad7 protein expression in HSCs. d) Fold changes of TGF $\beta$, T $\beta R$ I, T $\beta$ R II, smad3, smad4, smad7 expression from (c). Mean $\pm \mathrm{SD}, \mathrm{n}=3, * \mathrm{P}<0.01, \# \mathrm{P}<0.05$ vs Model group.

liver fibrosis through regulating TGF $\beta$ /smad signaling pathway.

\section{Chelerythrine inhibits the activation of hepatic stellate cells via TGFß1/smad signaling pathway}

To investigate how chelerythrine dose $(3.6 \mu \mathrm{M})$ regulates the TGF $\beta$ /smad signaling pathway to inhibit the activation of hepatic stellate cells, we analyzed the protein and mRNA expression of TGF $\beta 1$, T $\beta R I, T \beta R I I$, smad3, smad4, smad7. Western blotting and RT-PCR results suggested that chelerythrine concentration-dependently significantly down-regulated the protein and mRNA expression of TGF- $\beta 1$, T $\beta$ R II, and T $\beta$ RI in HSCs. Simultaneously, it also reduced the protein and mRNA expression of smad3, smad4 correspondingly. The protein expression of smad7 was obviously increased in the high chelerythrine dose $(6 \mu \mathrm{M})$, but it had no significant change in the low chelerythrine dose $(3 \mu \mathrm{M})$. As shown in Figure 7.

\section{DISCUSSION}

At present, there are no effective drugs for treating liver fibrosis (9-10). More effective and safe drugs for preventing and improving liver fibrosis are urgently needed. In this study, we demonstrated that chelerythrine has protective effects on liver fibrosis mice caused by carbon tetrachloride and common bile duct ligation.

Liver fibrosis is a repair response of the body to the inflammatory stimulation of chronic liver injury (11). The liver fibrosis model induced by intraperitoneal injection of $\mathrm{CCl}_{4}$ mixed solution is the most commonly used model of liver fibrosis. $\mathrm{CCl}_{4}$ is gradually metabolized in the endoplasmic reticulum to generate active trichloromethyl radicals and chlorine radicals, which cause degeneration and necrosis of liver cells, lyses lipid cell membranes in the liver, and a large number of intrahepatic enzymes (AST, ALT, etc.) leak out into the blood, its leakage content reflects the degree of liver damage to a certain 
extent, and long-term $\mathrm{CCl}_{4}$ stimulation can induce fibrotic damage(12-14).

Common bile duct ligation may induce liver fibrosis model in mice (15). The principle of this model is consistent with the clinical mechanism of secondary biliary cirrhosis. Ligation is the mechanical stimulation of the common bile duct, which causes extrahepatic biliary tract obstruction. This method has the characteristics of a less inflammatory response, rapid fibrosis formation, low spontaneous reversion rate, safety, non-toxicity, and low mortality. It is one of the ideal models for the pathophysiology and pharmacology of liver fibrosis (16-17).

AST and ALT are the active enzymes in the hepatocyte cytoplasm, which are the main indicators for evaluating liver function (18-19). Mild damage to the hepatocyte membrane can significantly increase the levels of AST and ALT in the blood. $\mathrm{In} \mathrm{CCI}_{4}$ and BDL model groups, serum AST and ALT were significantly increased, nevertheless, were decreased by chelerythrine. Hyaluronic acid (HA) is the main component of the glycosaminoglycan in the extracellular matrix, which can maintain the stability of the internal environment and the structure and function of liver cells. When liver fibrosis occurs, the content of Hyaluronic acid in the serum increased significantly. The content of serum Hyaluronic acid can reflect the degree of liver fibrosis (20). Hydroxyproline (Hyp) is a special amino acid of collagen, and its content can reflect the function of cell synthesis and secretion of collagen, and objectively reflect the degree of liver fibrosis (21). In our study, the content of HA and Hyp was significantly increased in two model groups and was obviously reduced in chelerythrine treatment groups. In the results of Sirus Red staining, the red area of collagen fibers in the administration groups was significantly decreased. The above results showed, chelerythrine inhibited $\mathrm{CCI}_{4}$ and BDL induced mice liver damage and improved mice liver fibrosis degree.

Both the $\mathrm{CCI}_{4}$ and BDL fibrosis model methods cause liver damage. When the liver is damaged, it may generate an oxidative stress reaction, which produces a large number of reactive oxygen species (ROS) (22-23). Recent studies have shown that ROS promoted TGF $\beta$ secretion (24). What's more, a large amount of TGF $\beta$ can induce hepatic stellate cells to transform from quiescent state to activated state, secreting plentiful extracellular matrix (ECM) and accelerating the process of liver fibrosis. MMP-2 plays a crucial role in the degradation of ECM, nevertheless, TIMP-1 facilitates ECM synthesis. Under normal physiological conditions, the production and degradation of ECM are in equilibrium. After the liver is damaged, the balance of production and degradation of ECM is broken.

In this report, in the model group $\left(\mathrm{CCI}_{4}\right.$ and $\mathrm{BDL})$, the concentration of ROS was significantly up-regulated, indicating that ROS played an important role in the formation of liver fibrosis. Correspondingly, there is a complex anti-oxidative stress system in mammals. Glutathione (GSH), possesses the functions of anti-oxidative stress, anti-inflammatory, detoxification, and regulating cell apoptosis. In our study, the level of GSH was lower in two model groups than control (sham) group. During the development of liver fibrosis, in order to repair oxidative stress, the consumption of GSH was increased. After treatment with chelerythrine, the level of ROS was obviously reduced, the level of GSH was significantly increased. It can be seen that chelerythrine improved liver cell damage, increased the level of serum GSH, and enhanced its ability to resist oxidative stress, thereby protecting the liver. However, its long-term protective effect on the liver needs further study.

In this study, the expression of MMP-2 was obviously decreased, whereas TIMP-1 expression was significantly increased in $\mathrm{CCI}_{4}$ and $\mathrm{BDL}$ model groups. Chelerythrine treatment distinctly decreased TIMP-1 expression and increased MMP-2 expression. The results indicated that chelerythrine prevented mice liver fibrosis by $\mathrm{CCI}_{4}$ and BDL through accelerating degradation and suppressing the synthesis of ECM.

Activated hepatic stellate cells are the cytological basis for hepatic fibrosis, and play an important role in the process of liver fibrosis (25-26). When the liver is affected by various pathogenic factors such as injury and inflammation, quiescent hepatic stellate cells are activated, which transform into myofibroblasts, which secrete a variety of pro-inflammatory and pro-fibrotic factors (27). The synthesis of $\alpha$-SMA increased and produced a large number of ECM components mainly composed of Col-I and Col-III, which were excessively deposited in the liver, further forming liver fibrosis (28). RT-PCR and western blotting showed that the expression of $\alpha$-SMA, Col-I was significantly increased in the $\mathrm{CCI}_{4}$ and BDL model groups. Then, chelerythrine can significantly reduce the expression of $\alpha$-SMA, Col-I in our research results. Taken together, chelerythrine can inhibit liver fibrosis by inhibiting the activation of hepatic stellate cells and ECM deposition.

When the liver is damaged, hepatic stellate cells are activated. In vitro, we detected the expression associated with hepatic stellate cell activation, 
including $\alpha$-SMA, Col-I, fibronectin. The results manifested that chelerythrine down-regulated obviously the protein and mRNA expression of $\alpha$-SMA, Col-I, fibronectin. In brief, chelerythrine can inhibit the activation of hepatic stellate cells and the production of ECM, then inhibiting liver fibrosis.

Liver fibrosis is a complex process. It is now widely accepted that TGF- $\beta 1$ is a major cytokine in the regulation of collagen protein synthesis, the production, degradation, and accumulation of ECM (29-30). In addition, smads protein is an intracellular kinase substrate of the TGF- $\beta$ receptor, and a key protein for TGF- $\beta$ to enter the nucleus through cell membranes (31). Studies have shown that TGF- $\beta$ can bind to receptors, which leads to phosphorylation of $\operatorname{smad} 2 / 3$, and then forms a compound with smad4 to conduct from the cytoplasm into the nucleus, thereby binding to specific DNA sequences and regulating specific target genes transcription and highly expressing, especially ECM, to form liver fibrosis. Smad7 is a negative regulator in the signal transduction pathway and can compete with smad4 to bind to phosphorylated receptors, thereby hindering signal transduction. Mechanistically, TGF- $\beta / \mathrm{smad}$ signaling pathway is the main signal transduction pathway, simultaneously, playing an important role during liver fibrosis (32-34). In this study, chelerythrine significantly down-regulated the protein and mRNA expression of TGF $\beta$, T $\beta$ R I, T $\beta$ R II, consistently decreasing the protein and mRNA smad 3 and smad4 in $\mathrm{CCI}_{4}$ and BDL model mice. Similar results were obtained in TGF $\beta$ activated mouse hepatic stellate cells. The above results indicated chelerythrine inhibited liver fibrosis through the TGF $\beta 1 /$ smad signaling pathway.

In summary, after the liver is damaged, on the one hand, the liver quickly initiates an oxidative stress response, producing reactive oxygen species (ROS), which simulate TGF $\beta$ secretion of causing fibrosis factor, further leading to ECM deposition. On the other hand, a hepatic stellate cell is activated, which generates type I and III collagen and fibronectin, leading to liver fibrosis. In our study, chelerythrine concentration dependently increased the level of GSH, suppressed ECM deposition in two different models of experimental hepatic fibrosis, consistently, inhibited activation of hepatic stellate cells. Mechanically, chelerythrine increased the level of GSH, restrained TGF $\beta$, T $\beta$ R I, T $\beta$ R II, and smad family gene expression. In brief, chelerythrine prevented TGF $\beta /$ smad signaling pathway, inhibiting oxidative stress.

In conclusion, liver fibrosis is a complicated pathological process, in our study, the results support our hypothesis and provide novel insight into the mechanisms of chelerythrine in the protection of the liver, it may be one of the candidates for anti-liver fibrosis.

\section{Acknowledgments}

This work was supported by the Science and Technology Bureau of Qiqihar City, Heilongjiang Province, China, under Grant (LHYD-2021003); Clinical Research Fund Project of Qiqihar Academy of Medical Sciences(QMSI2020L-03); Basic Scientific Research Business Expenses of Heilongjiang Province, China, under Grant (2018-KYYWF-0099).

\section{Funding information}

This work was supported by the Science and Technology Bureau of Qiqihar City, Heilongjiang Province, China, under Grant (LHYD-2021003); Clinical Research Fund Project of Qiqihar Academy of Medical Sciences(QMSI2020L-03); Basic Scientific Research Business Expenses of Heilongjiang Province, China, under Grant (2018-KYYWF-0099).

\section{Conflict of interest}

The authors declare no conflict of interest.

\section{REFERENCES}

1. Kim J.Y., An H.J., Kim W.H., Gwon M.G., Gu H.: Mol. Ther. Nucleic Acids 8, 250 (2017).

2. Zou G.L., Zuo S., Lu S., Hu R.H., Lu Y.Y., et al.: World J. Gastroenterol. 25, 4222 (2019).

3. Dhar D., Baglieri J., Kisseleva T., Brenner D.A.: Exp. Biol. Med. (Maywood) 245, 96 (2020).

4. Tang L.-Y., Heller M., Meng Z., Yu L.-R., Tang Y., et al.: J. Biol. Chem. 292, 4302 (2017).

5. Fabregat I., Caballero-Diaz D.: Front. Oncol. 8, 357 (2018).

6. Meng X.M., Nikolic-Paterson D.J., Lan H.Y.: Nat. Rev. Nephrol. 12, 325 (2016).

7. Sitanggang E.J., Antarianto R.D., Jusman S.W.A., Pawitan J.A., Jusuf A.A.: Int. J. Stem Cells 10, 69 (2017).

8. Liu X.W., Tang C.L, Zheng H., Wu J.X., Wu F., et al.: J. Pharm. Biomed. Anal. 159, 252 (2018).

9. Chen D.-Q., Feng Y.-L., Cao G., Zhao Y.-Y.: Trends Pharmacol. Sci. 39, 937 (2018).

10. Roehlen N., Crouchet E., Baumert T.F.: Cells 9, 43 pages (2020). 
11. Poole L.G., Pant A., Cline-Fedewa H.M., Williams K.J., Copple B.L., et al.: Res. Pract. Thromb. Haemost. 4, 906 (2020).

12. Ma X., Luo Q., Zhu H., Liu X., Dong Z., et al.: J. Cell. Mol. Med. 22, 3965 (2018).

13. Wang R., Song F., Li S., Wu B., Gu Y., Yuan Y.: Drug Des. Devel. Ther. 13, 1889 (2019).

14. Wu H., Chen G., Wang J., Deng M., Yuan F., Gong J.: Cell Prolif. 53, 13 pages (2020).

15. Wei Z., Zhao D., Zhang Y., Chen Y., Zhang S., et al.: Biochem. Biophys. Res. Commun. 519, 854 (2019).

16. Tag C.G., Sauer-Lehnen S., Weiskirchen S., Borkham-Kamphorst E., Tolba R.H., et al.: J. Vis. Exp. 2015, 10 pages (2015).

17. Shafie F., Nabavizadeh F., Shafie Ardestani M., Panahi M., Adeli S., et al.: Can. J. Physiol. Pharmacol. 97, 691 (2019).

18. Wu L., Zhang Q., Mo W., Feng J., Li S., et al.: Sci. Rep. 7, 9289 (2017).

19. Zhang D., Hao X., Xu L., Cui J., Xue L., Tian Z.: Oncol. Lett. 14, 4511 (2017).

20. Qi X., Liu X., Zhang Y., Hou Y., Ren L., et al.: Med. Sci. Monit. 22, 2720 (2016).

21. Hu C., Zhao L., Tao J., Li L.: J. Cell. Mol. Med. 23, 7151 (2019).

22. El Awdan S.A., Abdel Rahman R.F., Ibrahim H.M., Hegazy R. R., El Marasy S.A., et al.: PLoS One 14, e0216301 (2019).
23. George J., Tsuchishima M., Tsutsumi M.: Cell Death Dis. 10, 18 (2019).

24. Lestari N., Louisa M., Soetikno V., Suwana A.G., Ramadhan P.A., et al.: J. Toxicol. 2018, 9 pages (2018).

25. Higashi T., Friedman S.L., Hoshida Y.: Adv. Drug Deliv. Rev. 121, 27 (2017).

26. Ling L., Li G., Wang G., Meng D., Li Z., Zhang C.: Mol. Med. Rep. 20, 1605 (2019).

27. Xu T., Lu Z., Xiao Z., Liu F., Chen Y., et al.: Cell Death Dis. 11, 11 pages (2020).

28. Shi H., Shi H., Ren F., Chen D., Chen Y., Duan Z.: J. Cell. Mol. Med. 21, 500 (2017).

29. Dewidar B., Meyer C., Dooley S., MeindlBeinker A.N.: Cells 8, 36 pages (2019).

30. Chan Y.T., Wang N., Tan H.Y., Li S., Feng Y.: Front. Pharmacol. 11, 22 pages (2020).

31. Zhang Y., Guo J., Li Y., Jiao K., Zhang Y.: Exp. Ther. Med. 17, 3935 (2019).

32. Dattaroy D., Seth R.K., Sarkar S., Kimono D., Albadrani M., et al.: Eur. J. Pharmacol. 841, 33 (2018).

33. Yang H.Y., Kim K.S., Lee Y.H., Park J.H., Kim J.-H., et al.: Int. J. Biol. Sci. 15, 800 (2019).

34. Yang Y.Z., Zhao X.J., Xu H.J., Wang S.C., Pan Y., et al.: Acta Pharmacol. Sin. 40, 879 (2019). 